\title{
Integrative radiogenomic profiling of squamous cell lung cancer
}

\author{
Mohamed E. Abazeed ${ }^{\star}, 1$, Drew J. Adams ${ }^{\star}, 2$, Kristen E. Hurov ${ }^{3}$, Pablo Tamayo ${ }^{4}$, Chad J. \\ Creighton $^{5}$, Dmitriy Sonkin ${ }^{3}$, Andrew O. Giacomelli ${ }^{6}$, Charles $\mathrm{Du}^{7}$, Daniel F. Fries ${ }^{8}$, Kwok- \\ Kin Wong 8,9 , Jill P. Mesirov ${ }^{4}$, Jay S. Loeffler ${ }^{10}$, Stuart L. Schreiber ${ }^{2,11,12}$, Peter S. \\ Hammerman $4,8, \S$, and Matthew Meyerson $4,8,13, \S$ \\ ${ }^{1}$ Harvard Radiation Oncology Program, Boston, MA 02215 \\ ${ }^{2}$ Chemical Biology Program, Broad Institute, Cambridge, MA 02142 \\ ${ }^{3}$ Novartis Institute for Biomedical Research, Cambridge, MA 02139 \\ ${ }^{4}$ The Broad Institute of MIT and Harvard, Cambridge, MA 02142 \\ ${ }^{5}$ Department of Medicine, Baylor College of Medicine, Houston, Texas 77030 \\ ${ }^{6}$ Harvard Medical School, Boston, MA 02215 \\ ${ }^{7}$ Harvard University, Cambridge, MA 02142 \\ ${ }^{8}$ Department of Medical Oncology, Dana-Farber Cancer Institute, Boston, Massachusetts 02215 \\ ${ }^{9}$ Belfer Institute for Applied Cancer Science, Dana-Farber Cancer Institute, Boston, MA 02214 \\ ${ }^{10}$ Department of Radiation Oncology, Massachusetts General Hospital and Harvard Medical \\ School, Boston, MA 02114 \\ ${ }^{11}$ Department of Chemistry and Chemical Biology, Harvard University, Cambridge, MA 02138 \\ ${ }^{12}$ Howard Hughes Medical Institute, Broad Institute, Cambridge, MA 02142 \\ ${ }^{13}$ Department of Pathology, Brigham and Women's Hospital, Boston, Massachusetts 02215
}

\section{Abstract}

Radiation therapy is one of the mainstays of anti-cancer treatment, but the relationship between the radiosensitivity of cancer cells and their genomic characteristics is still not well-defined. Here we report the development of a high-throughput platform for measuring radiation survival in vitro and its validation by comparison to conventional clonogenic radiation survival analysis. We combined results from this high-throughput assay with genomic parameters in cell lines from squamous cell lung carcinoma, which is standardly treated by radiation therapy, to identify parameters that predict radiation sensitivity. We showed that activation of NFE2L2, a frequent event in lung squamous cancers, confers radiation resistance. An expression-based, in silico screen nominated inhibitors of PI3K as NFE2L2 antagonists. We showed that the selective PI3K inhibitor, NVP-BKM120, both decreased NRF2 protein levels and sensitized NFE2L2 or KEAP1 mutant cells to radiation. We then combined results from this high-throughput assay with singlesample gene set enrichment analysis (ssGSEA) of gene expression data. The resulting analysis

\footnotetext{
§To whom correspondence should be addressed: Matthew Meyerson, Dana-Farber Cancer Institute, Phone: 617-632-4768, Dana Bldg, Rm. 1540, Fax: 617-582-7880, 44 Binney Street, 1523A, matthew_meyerson@dfci.harvard.edu, Boston, MA 02215, Peter Hammerman, Dana-Farber Cancer Institute, Phone: 617-632-4768, Dana Bldg, Rm. 1520, Fax: 617-582-7880, 44 Binney Street, $1523 \mathrm{~A}$, phammerman@ partners.org, Boston, MA 02215.

* These authors contributed equally to this work.

Conflict of interest: M.M. is a consultant to Novartis and receives research support from Novartis, and is a founding advisor and consultant to, and an equity holder in, Foundation Medicine. P.S.H. reports consulting fees from ARIAD.
} 
identified pathways implicated in cell survival, genotoxic stress, detoxification, and innate and adaptive immunity as key correlates of radiation sensitivity. The integrative, high-throughput methods shown here for large-scale profiling of radiation survival and genomic features of solidtumor derived cell lines should facilitate tumor radiogenomics and the discovery of genotypeselective radiation sensitizers and protective agents.

\section{Keywords}

TP53; cmap; radiomodifier

\section{INTRODUCTION}

The use of a combination of radiation and chemotherapy, or chemoradiation, is the mainstay of treatment for many solid malignancies (1). In recent years, the use of cancer chemotherapeutic drugs has increasingly been driven by genomic characteristics, especially for targeted therapies (2-4). In contrast, despite correlative studies that have established gene classifiers predictive of radiation response across the NCI-60 panel of cell lines (5-7), there have not been extensive systematic analyses of the correlation between radiation sensitivity and genomic parameters.

In 1956, Puck and Marcus described a technique for assessment of clonogenic growth of HeLa cells after exposure to radiation in vitro (8). The clonogenic assay is still widely considered the most reliable in vitro assay for assessing toxicity in cell lines, measuring the sum of all modes of cell death while simultaneously accounting for delayed growth arrest. Unlike cellular response to cytotoxic compounds, most cells lethally damaged by radiation do not immediately cease proliferation but may multiply for several generations before terminating reproduction (9). Therefore, short-term assays that are useful for the study of cytotoxic compounds have not proven effective in accurately profiling solid-tumor derived cell survival after exposure to radiation.

Although several high-throughput screening assays that measure cellular response to DNA double-strand breaks have been used effectively to identify modulators of DNA damage response (DDR) $(10,11)$, such pathway-focused assays lack the scope needed for a comprehensive evaluation of the physiological and genomic parameters influencing survival following exposure to radiation. The lack of a high-throughput assay measuring clonogenic survival is a major obstacle in radiobiology research. Such an assay could facilitate largescale studies to identify predictive markers for tumor response to therapy and facilitate development of rational combinatorial (chemoradiation) treatment. Several radiosensitizing drugs are currently used clinically, but despite their demonstrated efficacy they have numerous shortcomings $(12,13)$. In particular, their efficacy and toxicity is likely to vary based on the genetic characteristics of individual tumors, significantly limiting their optimal use. Recent studies have identified frequent and targetable genomic alterations that are correlated with the likelihood of response to specific agents, particularly for lung cancer (24). Similar studies are desperately needed to discover promising targets for agents that increase the radiotherapeutic ratio.

Herein, we report on a high-throughput platform that measures radiation survival and leverages cancer genomic data to advance knowledge of radiation tumor biology and therapeutic possibilities. 


\section{METHODS}

\section{Cell culture and irradiation}

Lung SqCC cell lines from the Cancer Cell Line Encyclopedia (CCLE) were authenticated per CCLE protocol (14) and grown in recommended media supplemented with $10 \%$ fetal bovine serum (Benchmark, CA) and $100 \mathrm{U} / \mathrm{mL}$ Penicillin, $100 \mu \mathrm{g} / \mathrm{mL}$ of Streptomycin, and $292 \mu \mathrm{g} / \mathrm{mL}$ L-Glutamine (Corning, NY). All cultures were maintained at $37^{\circ} \mathrm{C}$ in a humidified 5\% $\mathrm{CO}_{2}$ atmosphere and tested to ensure absence of Mycoplasma. Plates were treated with $0 \mathrm{~Gy}$ (no radiation) or with $1-10 \mathrm{~Gy}$ of $\gamma$-radiation delivered at $0.91 \mathrm{~Gy} / \mathrm{min}$ with a ${ }^{137} \mathrm{Cs}$ source using a GammaCell 40 Exactor (Best Theratronics; Ontario, Canada).

\section{Clonogenic survival}

Cells were plated at appropriate dilutions, irradiated, and incubated for 10-14 days for colony formation. For chemical radiosensitization measurements, drug was added 24 hours prior to irradiation. Colonies were fixed in a solution of acetic acid and methanol 1:3 (v/v) and stained with $0.5 \%(\mathrm{w} / \mathrm{v})$ crystal violet as previously described (15). A colony was defined to consist of 50 cells or greater. Colonies were counted digitally using ImageJ software as described (16). Integration of survival as a function of dose, or area under the curve, was calculated using Prism, GraphPad Software (La Jolla, CA).

\section{High-throughput proliferation assay}

Cells were plated using a Multidrop Combi liquid handler (Thermo Fisher) in quadruplicates for each time point at four cell densities (range 25-300 cells/well) in a white 384-well plate (Corning, NY). Plates were irradiated and at each time point, media was aspirated and $40 \mu \mathrm{L}$ of CellTiter-Glo® reagent (50\% solution in PBS) (Promega, WI) was added to each well. Relative luminescence units were measured using an Envision multilabel plate reader (Perkin Elmer) with a measurement time of 0.1 seconds. Luminescence signal is proportional to the amount of ATP present.

\section{Antibody and reagents}

Anti-NRF2 antibody (ab31163) was from Abcam (Cambridge, MA) (17). NVP-BKM120 and TGX-221 was from Selleck (Houston, TX). LY 294002 was from Cell Signaling Technology (Beverly, MA).

\section{RNA interference analysis and generation of LC-1/SQSF, hNQ01-ARE-luc}

Construction of the retroviral expression vector of short hairpin RNA (shRNA) was carried out as described (18). The shRNA-targeted sequences were as follows: NRF2-1 shRNA, AGAGCAAGATTTAGATCATTT; NRF2-2 shRNA, GCTCCTACTGTGATGTGAAAT. The control vector contains nontargeting shRNA sequence. The entry vectors were recombined with pLKO-Tet-ON-puro by LR reactions (Invitrogen, Carlsbad, CA), in accordance with the manufacturer's instructions. After infection (multiplicity of infection > 1 ), cells were selected and maintained in the presence of $1 \mu \mathrm{g} / \mathrm{ml}$ puromycin.

LC-1/SQSF, hNQO1-ARE-luc cells were produced as follows. hQR41-ARE sequence (19) and firefly luciferase-PEST sequence (Promega, WI) was cloned into plenti6/BLOCK-iTDEST lentivirus backbone (Invitrogen, NY) at the restriction sites of NcoI and Hind III. The engineered region was sequence verified and matched $100 \%$ of the expected sequences. The plasmid was stably infected to the LC-1/SQSF cell line, selected and maintained in $5 \mu \mathrm{g} / \mathrm{ml}$ Blasticidin in the recommended media. 


\section{TP53 and NFE2L2 Pathway Signatures}

Gene transcription signature of pathways TP53 (or p53) and NFE2L2 (or NRF2) were defined as described (20) (see Supplementary Data).

\section{Single-sample GSEA and the information-based association metric}

The single-sample GSEA enrichment scores were obtained as described (see Supplementary Data).

\section{RESULTS}

\section{Development and validation of a high-throughput radiation survival assay}

To profile radiation response in lung $\mathrm{SqCC}$ cell lines, we performed clonogenic assays on 18 lines after exposure to $0,2,5$, or 8 Gy of $\gamma$-rays. LOU-NH91 and SK-MES- 1 were nonclonogenic and SW1573 had prohibitively low plating efficiencies. We were therefore able to analyze survival for 15 of the 18 available cell lines (Fig. S1 and Table S1).

We measured radiation response in the same 18 cell lines in a format amenable to highthroughput profiling. We first optimized growth measurements in 384-well plates. The linear range for proliferation as a function of cell density was determined for each cell line; representative plots and light microscopy images for LUDLU-1 and EBC-1 after incubation for 9 days are shown in Fig. S2A and S2C. Using cell densities in the linear range of plating, we assessed growth $(0 \mathrm{~Gy})$ and recovery of growth after exposure to a range of doses of radiation by plotting relative luminescence units (RLU) as a function of time (Fig. S2B).

The proliferating fraction (mean RLU at dose $x$ / mean RLU of control) was plotted as a function of dose at 9 days for all cell lines (Fig. S2D and Table S2). We next examined whether the high-throughput platform correlated with clonogenic survival following exposure to radiation. We assessed the extent of correlation between individual doses (for each cell line, $n \geq 2$ ). $R^{2}$ values were calculated using the average value for each cell line by comparing surviving fraction at dose $x(\mathrm{SF} x)$ with proliferating fraction at dose $x(\mathrm{PF} x)$, generating a correlation table across the different doses of radiation (Fig. 1A). These data indicate that high-throughput proliferation better approximates clonogenic assay measurements at doses greater than $2 \mathrm{~Gy}$. Among the PFx doses, $R^{2}$ values were highest for PF4. Therefore, the dose which best approximates clonogenic survival is within the $\mathrm{GI}_{50}$ range for all cell lines (3-5 Gy).

For each proliferation and clonogenic experiment performed, we integrated survival as a function of dose and generated values for each cell line. Mean integral survival for 15 cell lines (for each cell line, $n \geq 2$ ) was calculated and compared to values from the clonogenic assay (Fig. 1B). Proliferation and colony integral survival values were significantly correlated, with Pearson $r=0.90, R^{2}=0.80$, and $P<0.0001$. Linear regression showed a slope of $0.73 \pm 0.1$.

We next assessed correlation between clonogenic survival and the high-throughput platform as a function of time (Fig. 1C). The concordance between clonogenic survival and proliferation after radiation exposure is time dependent, reaching statistical significance on day 8 and achieving the highest correlation on day 9 (Fig. 1C).

In addition to squamous cell lung carcinoma cell lines, we assayed a broader diversity of lung cancer cell lines for radiation response and found that the results of the high-throughput platform shown here are broadly consistent with the literature across multiple lung cancer cell types $(21,22)$ (Fig. S3). Taken together, these results indicate that the high-throughput 
platform facilitates the profiling of cell lines for radiation response and, despite some differences between the two assays, closely approximates clonogenic survival by most radiation response parameters.

\section{NFE2L2 activation regulates radiation resistance in lung SqCC}

NFE2L2 and KEAP1 are key regulators of oxidative and electrophilic stress response (23, 24). Exposure to reactive oxygen species (ROS) directly modifies cysteine residues in KEAP1, leading to NRF2 stabilization and translocation into the nucleus. NRF2 is responsible for the activation of cytoprotective genes, including genes that scavenge ROS $(25,26)$. Indeed, NRF2 activity has been demonstrated to confer both radiation resistance and lower endogenous levels of ROS $(27,28)$.

The extent to which NRF2 mediates therapeutic resistance has yet to be fully assessed across a large panel of cell lines or in lung SqCC. We sought to assess NRF2's role as a regulator of radiation resistance in lung $\mathrm{SqCC}$ cell lines. We first computed a summary gene signature score for NRF2 activation (see Methods and Supplementary Data) for each cell line profiled and determined the extent of correlation with radiation response. Survival after radiation exposure was significantly correlated with NRF2 score, with Pearson $r=0.47, R^{2}=0.22$, and $P=0.047$ (Fig. 2A). Directed sequencing of NFE2L2 and KEAP1 revealed a high frequency of alterations in lung SqCC cell lines (Table S3). We then stratified radiation response by mutation and high NRF2 score, defined as a value greater than the median (0.10) (Fig. 2B). These data demonstrated that high NRF2 score is a good predictor of radiation resistance.

Disruption of the KEAP1 binding sites within NRF2 ( $\mathrm{L}_{23}$ WRQDIDLG and $\mathrm{D}_{77}$ EETGE) (Fig. 2C) have been previously shown to decrease NRF2-KEAP1 binding, inhibit KEAP1mediated degradation, and ultimately promote transcriptional activity of NRF2 (29). Consistent with these results, both NFE2L2 mutant LC-1/SQSF (NRF2 score, 0.27) and KEAP1 mutant HCC15 (NRF2 score, 0.26) cell lines showed greater NRF2 protein levels compared to NFE2L2/KEAP1 wild type cell line RERF-LC-AI (NRF2 score, 0.10) (Fig. 2D). The magnitude of NRF2 stabilization in LC-1/SQSF and HCC15 approximated that observed for RERF-LC-AI cells treated with tert-Butylhydroquinone ( $t \mathrm{BHQ}$ ), a chemical promoter of NRF2 stabilization and transcriptional activity (30).

To determine the role of NRF2 in regulating radiation response, we established LC-1/SQSF cells that stably express shRNAs against NRF2. Two lentiviral-inducible NRF2-targeted shRNAs (shNRF2-1 and shNRF2-2) significantly reduced the expression of endogenous mutant NRF2 compared with control shNTC (Fig. 2E). We tested clonogenic survival after lentiviral-induction in cells that stably expressed shNTC, shNRF2-1, and shNRF2-2. Cells that stably expressed shNRF2-2 had significantly diminished clonogenic capacity, correlating with the extent of reduced NRF2 protein, and therefore could not be evaluated for radiation response (Fig. 2F). To determine whether NRF2 has a role in resistance to radiation, we treated shNTC and shNRF2-1 infected LC-1/SQSF cells with radiation and then examined their clonogenic survival. NRF2-downregulated cells were more sensitive to radiation than control cells (Fig. 2G). It is important to note that this experiment models single fraction treatment. Patients generally receive multiple fractions of treatment, with a predicted compounding of the sensitization effect.

To determine whether NRF2 has a role in radiation resistance in a cell line that does not contain activating mutations in NFE2L2 or KEAP1, we established RERF-LC-AI cells that stably express shRNAs against NRF2. shNRF2-1 had a minimal affect on NRF2 protein level while shNRF2-2 significantly reduced the expression of endogenous NRF2 compared with control shNTC (Fig. 2H). In contrast to the diminished clonogenic capacity and 
radiosensitivity phenotype observed in LC-1/SQSF, significant reduction of NRF2 in RERFLC-AI did not result in any observable decrease in clonogenic capacity or radiation sensitivity (Fig. 2I and 2J). Taken together, these data indicate that mutation and activation of the NFE2L2 pathway confers radiation resistance in lung SqCC.

To determine the extent of activation of NFE2L2 across multiple tumor types, we computed a summary gene signature score for NRF2 activation for each cell line in the CCLE (967 cell lines) (Fig. S4). This indicates that activation of NRF2 is not unique to specific tumor lineages and nominates it as radiotherapeutic target in a wide range of malignancies.

\section{PI3K inhibitors are NRF2 antagonists and radiation sensitizers}

Multiple in silico approaches of high-throughput discovery have emerged, including the Connectivity Map (cmap), a Web-based tool that comprises a large gene expression database generated from human cancer cell lines treated with different chemicals $(31,32)$. Several studies have demonstrated the utility of chemical genomics in modulating biological processes by querying gene expression patterns (33-38). We screened for compounds whose expression negatively correlates with the NFE2L2 signature. The results of the screen are listed by rank order using a matching algorithm based on the non-parametric rank-ordered Kolmogorov-Smirnov statistics transformed to a 'connectivity score' ranging from +1 to -1 (Fig. 3A). A negative score denotes a negative correlation between a query signature and an individual chemical, indicating that the PI3K inhibitors genistein, LY 294002, and wortmannin are putative NRF2 antagonists (see Fig. 3B for a "bar view" of individual cmap instances).

Expression of active PI3K has long been implicated in regulating therapeutic (chemical and radiation) resistance $(39,40)$, although the precise mechanism(s) of resistance remain poorly defined. NRF2 has previously been show to require cooperation from active PI3K-Akt signaling (41). These results, coupled with the in silico findings above, suggested that PI3KAkt inhibition may be an effective strategy to antagonize NRF2 and by extension effect radiosensitization. To test this, we first established LC-1/SQSF cells that stably express hNQO1-ARE-luc, containing cis-acting antioxidant response regulatory elements in the 5'flanking region of $\mathrm{NAD}(\mathrm{P}) \mathrm{H}$ :quinone oxidoreductase (NQO1) (19), a gene induced by NRF2. We measured luciferase activity in LC-1/SQSF, hNQO1-ARE-luc cells after incubation with LY 294002, NVP-BKM120, or TGX-221. The pan-PI3K inhibitors LY 294002 and NVP-BKM120, but not the PI3K $\beta$ selective inhibitor TGX-221, resulted in a significant decrement in NRF2 activity (top panel, Fig. 3C). We measured cellular viability after incubation with LY 294002, NVPBKM120, or TGX-221. The decrement in cellular viability at 48 hours (lower panel, Fig. 3C) mirrored the effect on NRF2 activity at 24 hours. This correlation between viability and NRF2 activity suggests that either LC-1/SQSF cells are dependent on NRF2 for viability (suggested by Fig. 2F) or that toxicity leads to a non-specific decrement in NRF2 activity.

To address this, we measured NRF2 protein level in the same cells after incubation with LY 294002, NVP-BKM120, or TGX-221 at 24 hours, an interval of time that showed a negligible decrement in viability (viability $>90 \%$ ). Consistent with the reporter assay, LY 294002 and NVP-BKM120, but not TGX-221, resulted in a significant decrement in NRF2 protein level (Fig. 3D). NVP-BKM120 resulted in a similar decrement in NRF2 protein level for HCC15 and RERF-LC-AI cells treated with $t \mathrm{BHQ}$. These results indicate that an active PI3K pathway is required for NRF2 stability and that the putative target for NRF2 antagonism is PI3K through isoforms other than $\mathrm{PI} 3 \mathrm{~K} \beta$.

Since NRF2 down-regulation effects radiosensitization in LC-1/SQSF cells (Fig. 2), and NVP-BKM120 results in NRF2 down-regulation, we predicted that NVP-BKM120 can 
function as a radiation sensitizer in cell lines with an active NRF2 pathway. LC-1/SQSF, HCC15, and A549 cells exposed to NVP-BKM120 and radiation showed a synergistic decrement in clonogenic survival compared to control (0 Gy) cells (Fig. 4). RERF-LC-AI and SQ-1 (NFE2L2 and KEAP1 wild type) cells showed significantly less synergy between NVP-BKM120 and radiation. These results indicate that treatments that antagonize NRF2mediated transcription can be potent radiation sensitizers and suggest that the synergy between PI3K inhibitors and radiation are greater in cell lines with an active NRF2 pathway.

\section{Gene expression analysis identifies pathways that correlate with radiation response in lung SqCC}

Susceptibility of tumors to radiation is regulated by several pathways mediating the cellular response to radiation-induced damage. To identify pathways that are differentially correlated with radiation response, we used ssGSEA projection (42) as a hypothesis-generating gene set identification tool.

ssGSEA identifies biologically relevant gene sets that correlate with a functional output by estimating the degree to which an established gene set is overrepresented at the (top or bottom) of the sorted gene expression values list in each sample. To achieve this, we calculated an ssGSEA enrichment score, which is based on the weighted difference of the Empirical Cumulative Distribution Functions of the genes in the set relative to the genes not included in an individual set (42). The result is a single score per cell line per gene set, transforming the original dataset into a more interpretable higher-level description. Gene sets were obtained from the C2 sub-collection of the Molecular Signatures database (MSigDB) (43), an additional collection of oncogenic signatures, and other cancer-related gene sets curated from the literature, resulting in a dataset that has 4,628 pathway profiles for each sample. The association between the ssGSEA profiles for each gene set and the radiation response profile is then determined using an information-based similarity metric (RNMI) (see Methods and Supplementary Data) and the dataset is resorted based on this metric to identify correlates and anti-correlates with radiation survival (Fig. S5 and S6).

We first compared the profiles of each gene set/pathway with the radiation response scores (integral survival by clonogenic assay and high-throughput platform). The ssGSEA scores are displayed in a heatmap with the top 15 gene sets that correlate and anti-correlate with radiation survival (Fig. S5 and Table S3). Analysis of the both the top 15 and top 50 gene sets (Fig. S5 and S6) revealed overlap in the gene sets identified by the clonogenic assay and high-throughput platform. Moreover, we inserted the calculated ssGSEA enrichment scores utilizing the NRF2 gene set and identified NRF2 activation as positively correlated with radiation resistance; the NRF2 gene set was ranked number 48 using survival data from the high-throughput platform. These results indicate that ssGSEA is a robust gene set identification tool.

An analysis of the gene sets that differentially correlated with radiation survival suggested that pathways implicated in cell survival, genotoxicity, detoxification and innate or adaptive immunity can regulate radiation response. Moreover, the identity of individual gene sets and their correlation with radiation response can identify and aid in the validation of targets for potential radiotherapeutic sensitization. For example, a TP53 gene set was identified by ssGSEA (rank number seven in the high-throughput platform analysis [Fig. S5 and S6]), suggesting that TP53 transcriptional activation mediates radiation resistance. Despite a well defined role for TP53 in regulation of DNA repair, cell cycle arrest, and apoptosis after genotoxic stress (44), the predictive role of TP53 mutations in radiation response has been uncertain. 
Beyond demonstrating a proof of the concept that ssGSEA can identify know regulators of radiation response, we sought to lend clarity to the role of alterations in TP53 on radiation response across a panel of cell lines. We first assessed TP53 mutations, LOH, and mRNA levels in the lung SqCC cell lines profiled for radiation response (Table S4). TP53 is frequently altered in the lung SqCC cell lines; eight of eighteen cell lines having missense mutations, all of which cluster in the DNA binding domain (aa. 101-305). We compared TP53 mRNA levels as a function of genotype and found a significant difference in mRNA levels in cell lines with non-disruptive (missense mutation and intronic insertion) versus disruptive (non-sense mutation and deletion) alterations $(P<0.0001)$ (Fig. S7A). We compared radiation response as a function of genotype (Fig. S7B); there was a statistically significant difference between cell lines with wild type and non-disruptive mutations $(P=$ $0.004)$ and between cell lines with disruptive and non-disruptive mutations $(P=0.01)$ in TP53. We plotted integral survival values derived from clonogenic (Fig. S7C) and highthroughput platform (Fig. S7D) as a function of TP53 mRNA level and determined statistically significant correlations, $R^{2}=0.57$ and $0.33, P=0.001$ and 0.01 , respectively. We computed a score for TP53 activation using a previously described gene signature independent of the gene set included in ssGSEA (see Methods) and compared TP53 signature scores with radiation response. High-throughput platform integral survival and TP53 score were significantly correlated, $R^{2}=0.28$, and $P=0.03$ indicating that a high TP53 signature score is a reasonable predictor of radiation response (Fig. S7E). Taken together, these data indicate that mutation and activation of the TP53 pathway is a significant predictor of radiation resistance in lung SqCC and highlights the utility of ssGSEA as a radiogenomic tool.

\section{DISCUSSION}

The clonogenic assay has long been considered the most reliable in vitro assay for measuring cell survival after exposure to radiation $(45,46)$. We have adapted the clonogenic assay to measure radiation response in high-throughput form. We reconstituted clonogenic growth by automated plating coupled with assay miniaturization into a 384-well per plate format. The platform accurately measured radiation survival in lung SqCC, closely approximating most radiation parameters obtained from clonogenic survival assays. It also appears to accurately profile lung cell lines from non-squamous lineages, suggesting that it can be applied broadly. It is not clear that measurement of proliferation at 9 days will be sufficient for the accurate profiling of all cell lines, especially those at the extreme ends of growth rates. Nonetheless, minor adaptations of the platform (ie plating density and/or time to readout) should allow for the accurate profiling of a wide range of cell lines.

Compared to lung adenocarcinoma, there have been very few therapeutic advances in lung $\mathrm{SqCC}$ (47). Recently, greater insight into the genomic landscape of lung SqCC has been achieved (20), suggesting marked genomic complexity with frequent alterations in TP53, $C D K N 2 A / R B 1, N F E 2 L 2 / K E A P 1 / C U L 3$, and PI3K/AKT. To leverage cancer genomic data to advance knowledge of radiation tumor biology and therapeutic possibilities, we profiled the majority of lung squamous cell lines available for study. We demonstrated that NFE2L2 mutations leading to pathway activation conferred radiation resistance. NFE2L2 pathway alterations are frequent occurrences in lung SqCC, found in $38 \%$ of the cell lines we analyzed and $34 \%$ of TCGA samples (20). In a demonstration of the potential of combining the profiling of cell lines for radiation survival with expression-based databases, we performed an in silico screen and identified PI3K inhibitors as NRF2 antagonists and radiation sensitizers. Taken together, our data suggest assessing these compounds clinically in genotype selected populations. 
We introduced the use of single-sample gene set enrichment analysis (ssGSEA) as a hypothesis-generating computational approach to search for genetic markers and cellular pathways that correlate with radiation response. We identified several pathways previously implicated in radiation response including pathways mediating cell survival, genotoxicity, detoxification and innate or adaptive immunity. In a demonstration of the utility of ssGSEA, we analyzed the role of TP53 mutation in regulating radiation response in lung SqCCs. We showed that TP53 missense mutation and pathway activation correlated with radiation resistance whereas TP53 disruptive mutations correlated with radiation sensitivity, a previously unappreciated distinction in lung SqCC. Moreover, these data are consistent with clinical outcomes. In patients with locally advanced NSCLC treated with radiation therapy alone, mutant TP53 (by directed sequencing of the DNA binding domain of the gene) resulted in a decreased treatment response (48-50).

In summary, we have developed a high-throughput platform that accurately measures radiation survival in vitro and significantly facilitates the study of tumor radiogenomics. We also incorporated ssGSEA and cmap for in silico high-throughput gene pathway and drug discovery. Together, these approaches outline a systematic and comprehensive strategy to identify key genetic correlates of radiation response and potent radiation modifiers whose effect is greatest in patients with specific genomic alterations.

\section{Supplementary Material}

Refer to Web version on PubMed Central for supplementary material.

\section{Acknowledgments}

M.E.A. was supported by NIH training grant 5T32CA009382-30 and by funding from the Harvard Radiation Oncology Program. M.M. is supported by NCI RO1 CA109038. P.S.H. is supported by the Stephen D. and Alice Cutler Investigator Fund and by K08 CA163677. We thank the Novartis Institute for Biomedical Research for support. The experiments described in Fig. S3 were supported in part by NIH RC2 CA138399-01 (S.L.S). We thank the NIGMS for partial support (GM38627, awarded to S.L.S.). S.L.S. is an Investigator with the Howard Hughes Medical Institute. We also thank Y. Yan-Neale, T. Chen, W. Shao, and C. Guy for generation of stable cell lines and for helpful discussions during the course of this work.

\section{REFERENCES}

1. Curran WJ. New chemotherapeutic agents: update of major chemoradiation trials in solid tumors. Oncology (Williston Park). 2002; 63(Suppl 2):29-38.

2. Hammerman PS, Janne PA, Johnson BE. Resistance to Epidermal Growth Factor Receptor Tyrosine Kinase Inhibitors in Non-Small Cell Lung Cancer. Clin Cancer Res. 2009; 15:7502-7509. [PubMed: 20008850]

3. Paez JG, Janne PA, Lee JC, Tracy S, Greulich H, Gabriel S, et al. EGFR mutations in lung cancer: correlation with clinical response to gefitinib therapy. Science. 2004; 304:1497-1500. [PubMed: 15118125]

4. Kwak EL, Bang YJ, Camidge DR, Shaw AT, Solomon B, Maki RG, et al. Anaplastic lymphoma kinase inhibition in non-small-cell lung cancer. N Engl J Med. 2010; 363:1693-1703. [PubMed: 20979469]

5. Amundson SA, Do KT, Vinikoor LC, Lee RA, Koch-Paiz CA, Ahn J, et al. Integrating global gene expression and radiation survival parameters across the 60 cell lines of the National Cancer Institute Anticancer Drug Screen. Cancer research. 2008; 68:415-424. [PubMed: 18199535]

6. Eschrich S, Zhang H, Zhao H, Boulware D, Lee JH, Bloom G, et al. Systems biology modeling of the radiation sensitivity network: a biomarker discovery platform. International journal of radiation oncology, biology, physics. 2009; 75:497-505. 
7. Torres-Roca JF, Eschrich S, Zhao H, Bloom G, Sung J, McCarthy S, et al. Prediction of radiation sensitivity using a gene expression classifier. Cancer research. 2005; 65:7169-7176. [PubMed: 16103067]

8. Puck TT, Marcus PI. Action of x-rays on mammalian cells. J Exp Med. 1956; 103:653-666. [PubMed: 13319584]

9. Eriksson D, Stigbrand T. Radiation-induced cell death mechanisms. Tumour Biol. 2010; 31:363372. [PubMed: 20490962]

10. Bardelle C, Boros J. Development of a high-content high-throughput screening assay for the discovery of ATM signaling inhibitors. Journal of biomolecular screening. 2012; 17:912-920. [PubMed: 22653913]

11. Tseng HM, Shum D, Bhinder B, Escobar S, Veomett NJ, Tomkinson AE, et al. A high-throughput scintillation proximity-based assay for human DNA ligase IV. Assay and drug development technologies. 2012; 10:235-249. [PubMed: 22192310]

12. Katz D, Ito E, Liu FF. On the path to seeking novel radiosensitizers. International journal of radiation oncology, biology, physics. 2009; 73:988-996.

13. Auperin A, Le Pechoux C, Rolland E, Curran WJ, Furuse K, Fournel P, et al. Metaanalysis of concomitant versus sequential radiochemotherapy in locally advanced non-small-cell lung cancer. J Clin Oncol. 2010; 28:2181-2190. [PubMed: 20351327]

14. Barretina J, Caponigro G, Stransky N, Venkatesan K, Margolin AA, Kim S, et al. The Cancer Cell Line Encyclopedia enables predictive modelling of anticancer drug sensitivity. Nature. 2012; 483:603-607. [PubMed: 22460905]

15. Franken NA, Rodermond HM, Stap J, Haveman J, van Bree C. Clonogenic assay of cells in vitro. Nat Protoc. 2006; 1:2315-2319. [PubMed: 17406473]

16. Cai Z, Chattopadhyay N, Liu WJ, Chan C, Pignol JP, Reilly RM. Optimized digital counting colonies of clonogenic assays using ImageJ software and customized macros: comparison with manual counting. International journal of radiation biology. 2011; 87:1135-1146. [PubMed: 21913819]

17. Pendyala S, Moitra J, Kalari S, Kleeberger SR, Zhao Y, Reddy SP, et al. Nrf2 regulates hyperoxiainduced Nox4 expression in human lung endothelium: identification of functional antioxidant response elements on the Nox4 promoter. Free radical biology \& medicine. 2011; 50:1749-1759. [PubMed: 21443946]

18. Narisawa-Saito M, Handa K, Yugawa T, Ohno S, Fujita M, Kiyono T. HPV16 E6- mediated stabilization of ErbB2 in neoplastic transformation of human cervical keratinocytes. Oncogene. 2007; 26:2988-2996. [PubMed: 17146442]

19. Moehlenkamp JD, Johnson JA. Activation of antioxidant/electrophile-responsive elements in IMR-32 human neuroblastoma cells. Archives of biochemistry and biophysics. 1999; 363:98-106. [PubMed: 10049503]

20. Hammerman PS, Lawrence MS, Voet D, Jing R, Cibulskis K, Sivachenko A, et al. Comprehensive genomic characterization of squamous cell lung cancers. Nature. 2012

21. Carmichael J, Degraff WG, Gamson J, Russo D, Gazdar AF, Levitt ML, et al. Radiation sensitivity of human lung cancer cell lines. Eur J Cancer Clin Oncol. 1989; 25:527-534. [PubMed: 2539297]

22. Duchesne GM, Eady JJ, Peacock JH, Pera MF. A panel of human lung carcinoma lines: establishment, properties and common characteristics. British journal of cancer. $1987 ; 56: 287$ 293. [PubMed: 2822071]

23. Itoh K, Chiba T, Takahashi S, Ishii T, Igarashi K, Katoh Y, et al. An Nrf2/small Maf heterodimer mediates the induction of phase II detoxifying enzyme genes through antioxidant response elements. Biochem Biophys Res Commun. 1997; 236:313-322. [PubMed: 9240432]

24. Itoh K, Wakabayashi N, Katoh Y, Ishii T, Igarashi K, Engel JD, et al. Keap1 represses nuclear activation of antioxidant responsive elements by Nrf2 through binding to the aminoterminal Neh2 domain. Genes Dev. 1999; 13:76-86. [PubMed: 9887101]

25. Jeyapaul J, Jaiswal AK. Nrf2 and c-Jun regulation of antioxidant response element (ARE)mediated expression and induction of gamma-glutamylcysteine synthetase heavy subunit gene. Biochemical pharmacology. 2000; 59:1433-1439. [PubMed: 10751553] 
26. Sjoberg L, Eriksen TE, Revesz L. The reaction of the hydroxyl radical with glutathione in neutral and alkaline aqueous solution. Radiat Res. 1982; 89:255-263. [PubMed: 6278530]

27. Singh A, Bodas M, Wakabayashi N, Bunz F, Biswal S. Gain of Nrf2 function in nonsmall- cell lung cancer cells confers radioresistance. Antioxid Redox Signal. 2010; 13:1627-1637. [PubMed: 20446773]

28. McDonald JT, Kim K, Norris AJ, Vlashi E, Phillips TM, Lagadec C, et al. Ionizing radiation activates the Nrf2 antioxidant response. Cancer Res. 2010; 70:8886-8895. [PubMed: 20940400]

29. Shibata T, Ohta T, Tong KI, Kokubu A, Odogawa R, Tsuta K, et al. Cancer related mutations in NRF2 impair its recognition by Keap1-Cul3 E3 ligase and promote malignancy. Proceedings of the National Academy of Sciences of the United States of America. 2008; 105:13568-13573. [PubMed: 18757741]

30. Kong AN, Owuor E, Yu R, Hebbar V, Chen C, Hu R, et al. Induction of xenobiotic enzymes by the MAP kinase pathway and the antioxidant or electrophile response element (ARE/EpRE). Drug Metab Rev. 2001; 33:255-271. [PubMed: 11768769]

31. Lamb J. The Connectivity Map: a new tool for biomedical research. Nat Rev Cancer. 2007; 7:5460. [PubMed: 17186018]

32. Lamb J, Crawford ED, Peck D, Modell JW, Blat IC, Wrobel MJ, et al. The Connectivity Map: using gene-expression signatures to connect small molecules, genes, and disease. Science. 2006; 313:1929-1935. [PubMed: 17008526]

33. Hassane DC, Guzman ML, Corbett C, Li X, Abboud R, Young F, et al. Discovery of agents that eradicate leukemia stem cells using an in silico screen of public gene expression data. Blood. 2008; 111:5654-5662. [PubMed: 18305216]

34. Hassane DC, Sen S, Minhajuddin M, Rossi RM, Corbett CA, Balys M, et al. Chemical genomic screening reveals synergism between parthenolide and inhibitors of the PI-3 kinase and mTOR pathways. Blood. 2010; 116:5983-5990. [PubMed: 20889920]

35. Hieronymus H, Lamb J, Ross KN, Peng XP, Clement C, Rodina A, et al. Gene expression signature-based chemical genomic prediction identifies a novel class of HSP90 pathway modulators. Cancer Cell. 2006; 10:321-330. [PubMed: 17010675]

36. Stegmaier K, Ross KN, Colavito SA, O'Malley S, Stockwell BR, Golub TR. Gene expressionbased high-throughput screening(GE-HTS) and application to leukemia differentiation. Nat Genet. 2004; 36:257-263. [PubMed: 14770183]

37. Stegmaier K, Wong JS, Ross KN, Chow KT, Peck D, Wright RD, et al. Signature-based small molecule screening identifies cytosine arabinoside as an EWS/FLI modulator in Ewing sarcoma. PLoS medicine. 2007; 4:e122. [PubMed: 17425403]

38. Wei G, Twomey D, Lamb J, Schlis K, Agarwal J, Stam RW, et al. Gene expression-based chemical genomics identifies rapamycin as a modulator of MCL1 and glucocorticoid resistance. Cancer Cell. 2006; 10:331-342. [PubMed: 17010674]

39. Gupta AK, Bakanauskas VJ, Cerniglia GJ, Cheng Y, Bernhard EJ, Muschel RJ, et al. The Ras radiation resistance pathway. Cancer Res. 2001; 61:4278-4282. [PubMed: 11358856]

40. McKenna WG, Muschel RJ, Gupta AK, Hahn SM, Bernhard EJ. The RAS signal transduction pathway and its role in radiation sensitivity. Oncogene. 2003; 22:5866-5875. [PubMed: 12947393]

41. Mitsuishi Y, Taguchi K, Kawatani Y, Shibata T, Nukiwa T, Aburatani H, et al. Nrf2 redirects glucose and glutamine into anabolic pathways in metabolic reprogramming. Cancer Cell. 2012; 22:66-79. [PubMed: 22789539]

42. Barbie DA, Tamayo P, Boehm JS, Kim SY, Moody SE, Dunn IF, et al. Systematic RNA interference reveals that oncogenic KRAS-driven cancers require TBK1. Nature. 2009; 462:108112. [PubMed: 19847166]

43. Available from: http://wwwbroadinstituteorg/msigdb/.

44. Freed-Pastor WA, Prives C. Mutant p53: one name, many proteins. Genes Dev. 2012; 26:12681286. [PubMed: 22713868]

45. Brown JM, Wouters BG. Apoptosis, p53, and tumor cell sensitivity to anticancer agents. Cancer research. 1999; 59:1391-1399. [PubMed: 10197600] 
46. Pomp J, Wike JL, Ouwerkerk IJ, Hoogstraten C, Davelaar J, Schrier PI, et al. Cell density dependent plating efficiency affects outcome and interpretation of colony forming assays. Radiother Oncol. 1996; 40:121-125. [PubMed: 8884965]

47. Perez-Moreno P, Brambilla E, Thomas R, Soria JC. Squamous cell carcinoma of the lung: molecular subtypes and therapeutic opportunities. Clin Cancer Res. 2012; 18:2443-2451. [PubMed: 22407829]

48. Hayakawa K, Mitsuhashi N, Hasegawa M, Saito Y, Sakurai H, Ohno T, et al. The prognostic significance of immunohistochemically detected $\mathrm{p} 53$ protein expression in non-small cell lung cancer treated with radiation therapy. Anticancer research. 1998; 18:3685-3688. [PubMed: 9854477]

49. Langendijk H, Thunnissen E, Arends JW, de Jong J, ten Velde G, Lamers R, et al. Cell proliferation and apoptosis in stage III inoperable non-small cell lung carcinoma treated by radiotherapy. Radiother Oncol. 2000; 56:197-207. [PubMed: 10927139]

50. Matsuzoe D, Hideshima T, Kimura A, Inada K, Watanabe K, Akita Y, et al. p53 mutations predict non-small cell lung carcinoma response to radiotherapy. Cancer Lett. 1999; 135:189-194. [PubMed: 10096428] 
a.

\begin{tabular}{c|cccccccc}
$R^{2}$ & PF1 & PF2 & PF3 & PF4 & PF5 & PF6 & PF8 & PF10 \\
\hline SF2 & 0.14 & 0.30 & 0.32 & 0.37 & 0.18 & 0.17 & 0.10 & 0.11 \\
SF5 & 0.60 & 0.77 & 0.84 & 0.87 & 0.78 & 0.72 & 0.48 & 0.13 \\
SF8 & 0.44 & 0.31 & 0.43 & 0.59 & 0.54 & 0.52 & 0.38 & 0.13
\end{tabular}

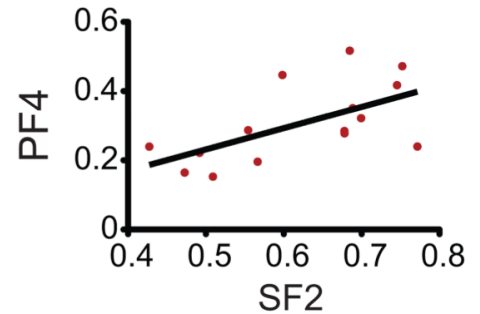

b.

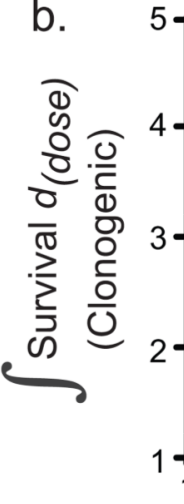

C.

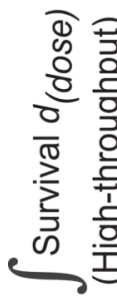

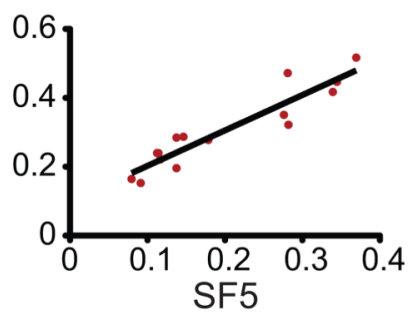

SF5

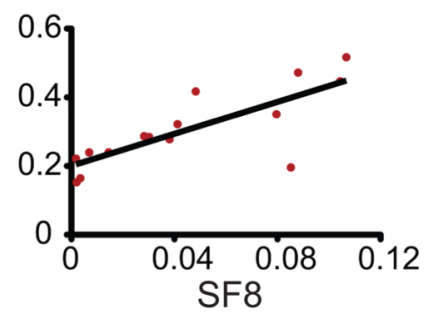

SF8

$\triangle \mathrm{LK}-2$

$\square \mathrm{EBC}-1$

$\triangle \mathrm{SQ}-1$

$\nabla$ HARA

$\checkmark$ CALU-1

O HCC 15

ㅁ $\mathrm{H} 2170$

․ H520

$\nabla$ HCC95

$\diamond \mathrm{H} 226$

$\triangle$ SW900

$\star$ LC-1/SQSF

A LUDLU-1

$\times$ RERF-LC-AI

$\odot$ KNS-62

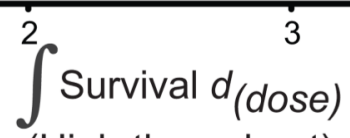

(High-throughput)

\begin{tabular}{c|ccc} 
& Pearson $r$ & $R^{2}$ & $P$ \\
\hline Day 4 & 0.44 & 0.19 & 0.10 \\
Day 6 & 0.21 & 0.05 & 0.44 \\
Day 8 & 0.72 & 0.53 & 0.002 \\
Day 9 & 0.90 & 0.80 & $<0.0001$
\end{tabular}

$\begin{array}{lllllllllllllllllllllllllll}2.0 & 2.5 & 3.0 & 3.5 & 4.0 & 4.5 & 2.0 & 2.5 & 3.0 & 3.5 & 4.0 & 4.5 & 2.0 & 2.5 & 3.0 & 3.5 & 4.0 & 4.5 & 2.0 & 2.5 & 3.0 & 3.5 & 4.0 & 4.5\end{array}$

\section{$\int \begin{gathered}\text { Survival } d(\text { dose }) \\ \text { (Clonogenic) }\end{gathered}$}

Fig. 1.

The high-throughput platform accurately profiles lung squamous cancer cell lines. (a) (Top) $R^{2}$ values were calculated comparing the proliferating fraction PF $x$ from high-throughput profiling and SFx from clonogenic assay. $R^{2}$ values depicted in red if $P<0.05$. (Bottom) Scatter plots and linear regression for PF4 with SF2, SF5, or SF8. (b) Integral survival was calculated for each cell line, $n \geq 2$ ). Error bars represent SEM. (c) Integral survival was calculated for proliferation assays for each cell line at days 4, 6, 8, and 9. Separately, integral survival was calculated for clonogenic survival assay. Data represents the average integral survival value for each cell line, $n \geq 2$. 


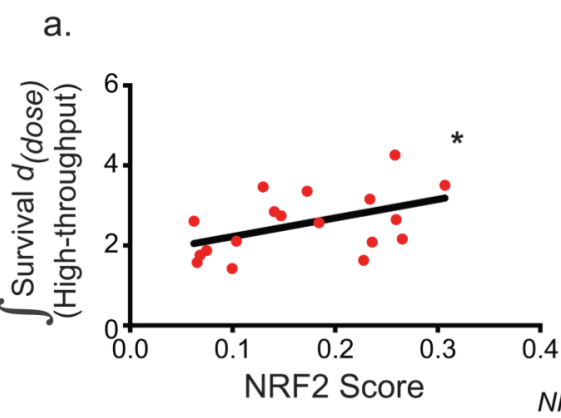

C.

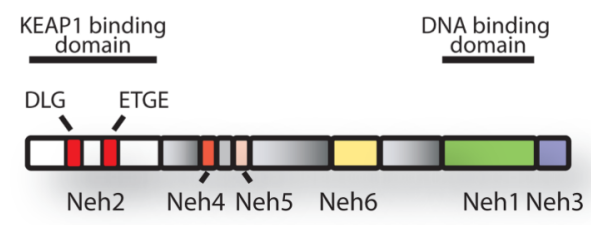

b.

b.

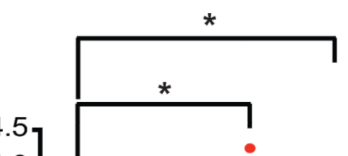

FE2L2/KEAP1 alteration: -+ NRF2 Score > 0.10:

d.

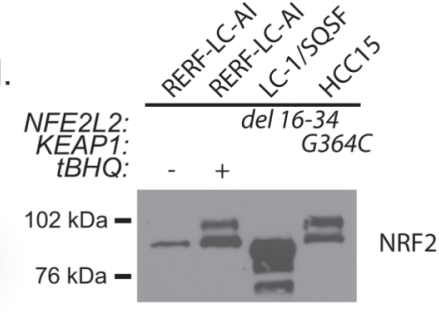

f.

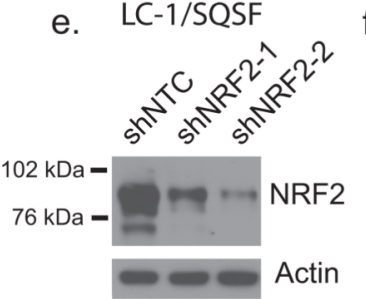

h.

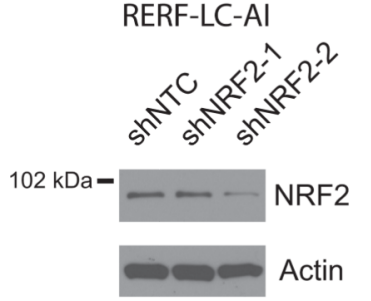

i.
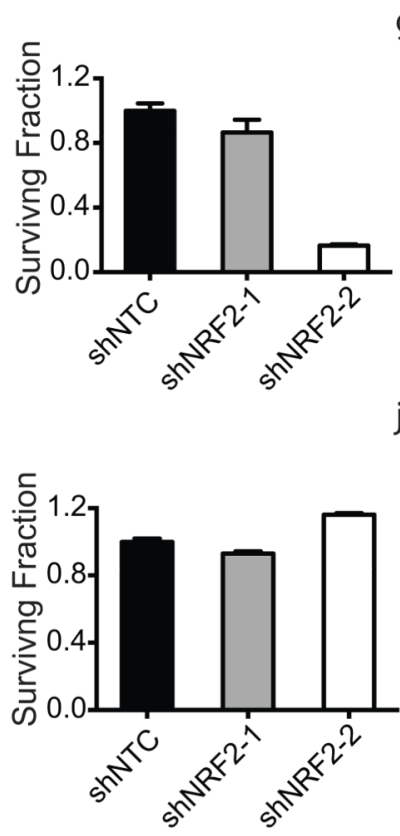

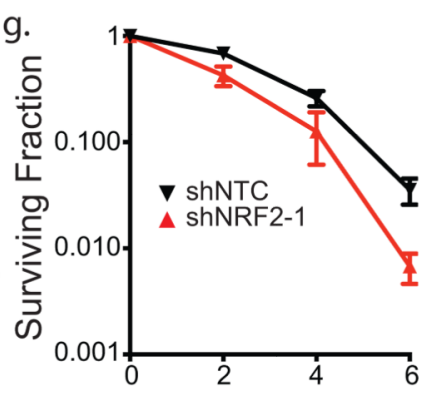

j.

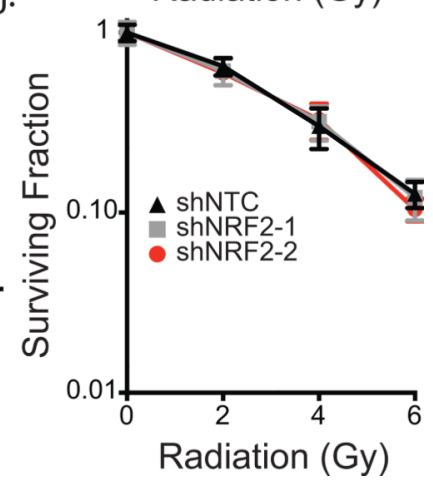

Fig. 2.

$N F E 2 L 2$ activation regulates radiation resistance and is a target for radiotherapeutic sensitization. (a) NRF2 scores from lung SqCC cell lines and integral survival were plotted. (b) Average integral survival values calculated from high-throughput assays were plotted as function of high NRF2 score defined as greater than the median $(0.10)$ and/or the presence of alteration in the coding region of NFE2L2 or KEAP1. (c) Schematic depiction of the functional domains of NRF2. The Neh2 domain contains the two KEAP1 association motifs, DLG and ETGE. (d) NRF2 protein level in cell lines: RERF-LC-AI (-/+ tBHQ), LC-1/ SQSF, and HCC15; Actin was used as a loading control. Immunblot analysis of NRF2 protein in LC-1/SQ-SF (e) and RERFLC-AI (h) cells infected with control shRNA (shNTC) 
and shNRF2-1 and shNRF2-2 after induction with Doxycycline for 24 hours; Actin was used as a loading control. LC-1/SQSF (f) and RERF-LC-AI (i) clones infected with shNTC, shNRF2-1, or shNRF2-2 were measured for clonogenic survival after induction with Doxycycline. LC-1/SQSF clones infected with shNTC or shNRF2-1 (g) and RERF-LC-AI clones infected with shNTC, shNRF2-1, or shNRF2-2 (j) were treated as control (0 Gy) or with radiation $(2,4,6$ Gy) after induction with Doxycycline for 24 hours. Data points represent mean values of duplicates (clonogenic survival) or six replicates (clonogenic survival after radiation) and are representative of three independent experiments. Error bars represent SEM. Cropped blots were imported directly into Adobe Illustrator CS6; no adjustments of brightness, contrast, or color balance were applied. 
b.

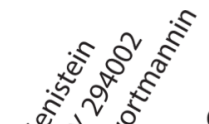

\section{a.

\begin{tabular}{ccc} 
Rank & cmap name & Score \\
\hline 1 & emetine & -1 \\
2 & cephaeline & -0.979 \\
3 & cephaeline & -0.967 \\
4 & cephaeline & -0.961 \\
5 & cephaeline & -0.96 \\
6 & ozagrel & -0.956 \\
7 & emetine & -0.956 \\
8 & homatropine & -0.954 \\
9 & genistein & -0.952 \\
10 & quinpirole & -0.951 \\
11 & LY294002 & -0.95 \\
12 & wortmannin & -0.946 \\
13 & rapamycin & -0.945 \\
14 & monorden & -0.944
\end{tabular}

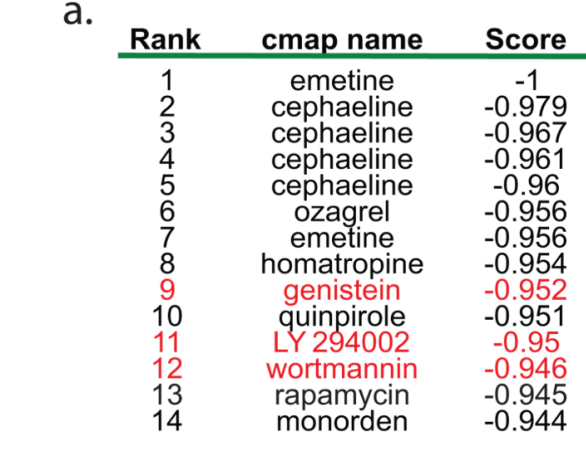

d.

\author{
LC- $1 /$ SQSF \\ (NFE2L2 mutant)
}
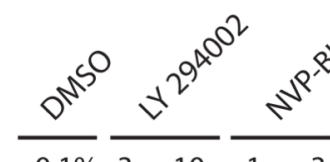

b.
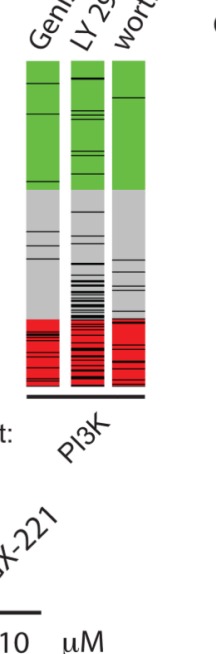

Targe

$$
\overline{0.1 \%} \overline{3} 10 \frac{}{1} 33 \quad 310
$$

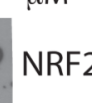

NRF2 $102 \mathrm{k}$

Actin

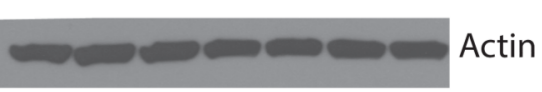

C.

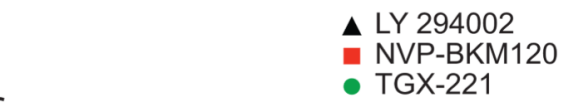

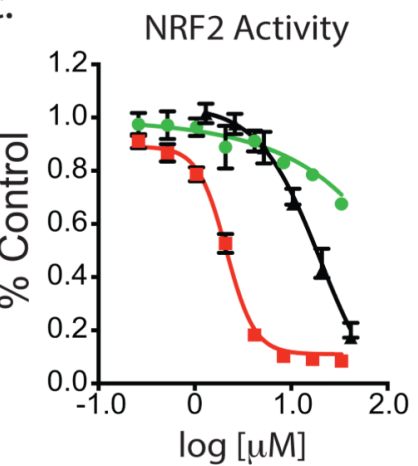

HCC 15

(KEAP1 mutant)

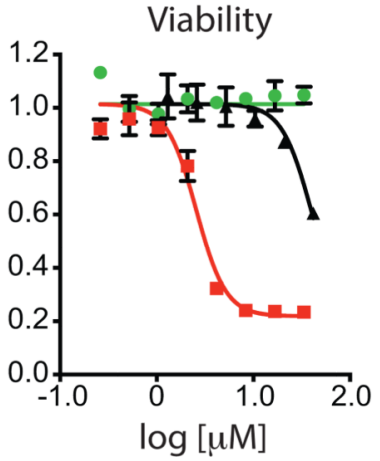

RERF-LC-AI

(NFE2L2 wild type)

NVP-BKM 120
NVP-BKM 120

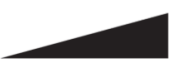

$\mu \mathrm{M} \quad \mathrm{tBHQ}$ :

0.10 .31

NRF2 $102 \mathrm{kDa}-$

$-++++$

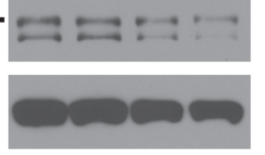

Actin

Fig. 3.

Inhibition of PI3K antagonizes NRF2. (a) The rank, cmap name, connectivity score for each of the selected chemicals is shown. (b) The "barview' is constructed from horizontal lines, each representing an individual treatment instance, ordered by their corresponding connectivity scores with the NFE2L2 signature (+1, top; -1 , bottom). All instances in the data set are colored in black. Colors applied to the remaining instances reflect the sign of their scores (green, positive; gray, null; red, negative). (c) LC-1/SQSF clones containing the ARE luciferase reporter were treated with LY 294002, NVP-BKM 120, and TGX-221 for 24 hours. Cellular viability was measured at 48 hours. Data points represent mean values of triplicates and error bars represent SD. The experiment was performed three times with comparable results. (d) The pan-PI3K inhibitor NVP-BKM 120 decreases NRF2 protein level. LC-1/SQSF cells were treated with control (DMSO), LY 294002, NVP-BKM 120, or TGX-221 and HCC15 cells were treated with DMSO and NVP-BKM120 for 24 hours and lysates were subjected to immunoblot analysis for the NRF2 protein. RERF-LC-AI cells were treated with DMSO and NVP-BKM120 for 24 hours followed by induction with 10 $\mu \mathrm{M}$ tBHQ for 24 hours before lysates were subjected to immunoblot analysis for the NRF2 protein. Actin was used as a loading control. Cropped blots were imported directly into Adobe Illustrator CS6; no adjustments of brightness, contrast, or color balance were applied. 

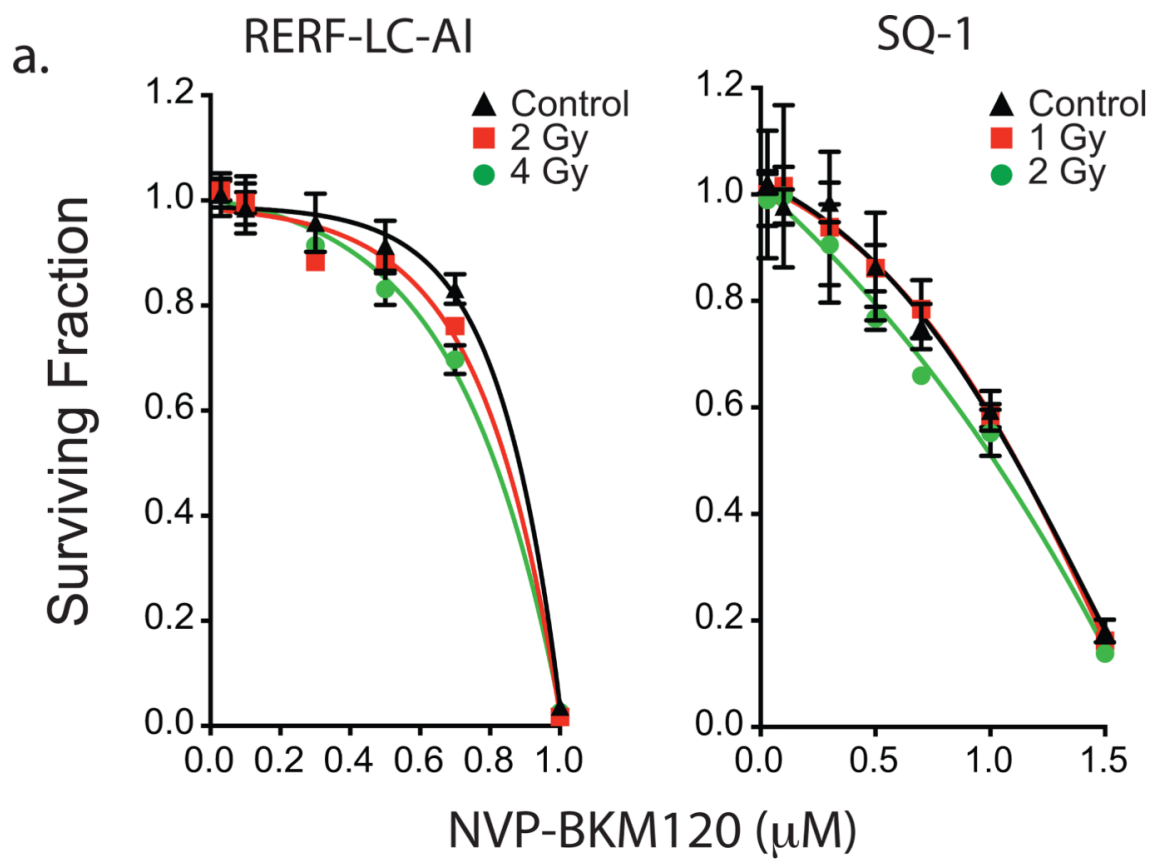

b.

a.

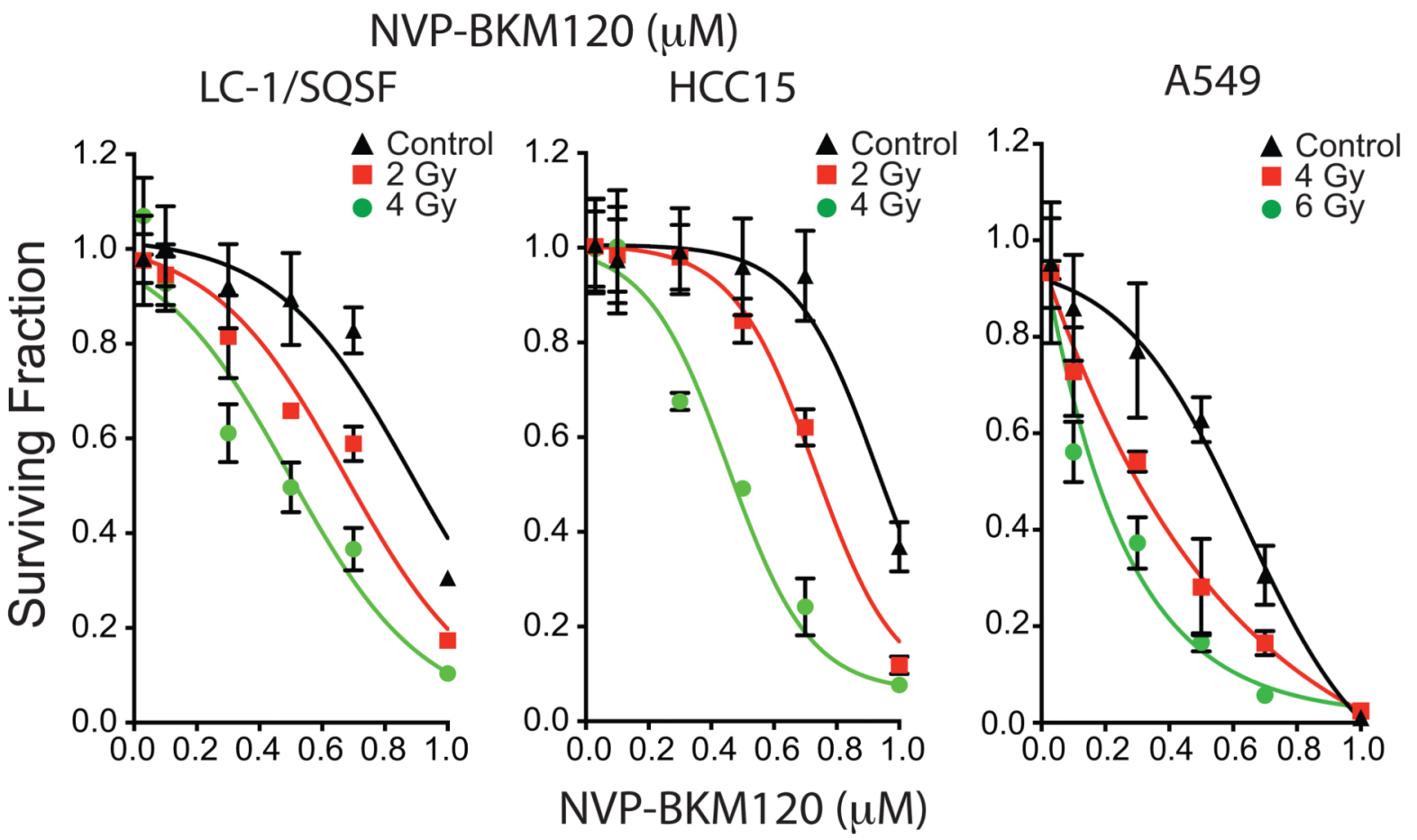

Fig. 4.

Inhibition of PI3K effects radiosensitization in cell lines with a NFE2L2 pathway alteration. (a) RERF-LC-AI, SQ-1, LC-1/SQSF, HCC15, or A549 cells were incubated with NVPBKM120 for 24 hours and treated as control (0 Gy) or with radiation. Survival is measured by clonogenic assay. Data points represent mean values of duplicates and error bars represent SD. The experiment was performed three times with comparable results. In the experiment shown in panel (a), surviving fraction after exposure to 2 and 4 Gy (RERF-LCAI, LC-1/SQSF, and HCC-15), 4 and 6 Gy (A549), and 1 and 2 Gy (SQ-1) radiation for cells incubated with DMSO alone are as follows: LC-1/SQSF, $0.71 \pm 0.11$ and $0.37 \pm 0.08$; HCC $15,0.75 \pm 0.9$ and $0.42 \pm 0.12$; RERF-LC-AI, $0.52 \pm 0.06$ and $0.33 \pm 0.01 ; \mathrm{A} 549,0.46$ 
\pm 0.09 and $0.24 \pm 0.06$; SQ-1, $0.65 \pm 0.16$ and $0.48 \pm 0.06$. (b) NFE2L2/KEAP1 genotype of cell lines tested for radiosensitization. 